\author{
Sebastião Iberes Lopes Melo ${ }^{1}$ \\ Juliane de Oliveira ${ }^{1}$ \\ Roberta Castilhos Detânico ${ }^{1}$ \\ Rudnei Palhano ${ }^{1}$ \\ Renata Marcelino Schwinden ${ }^{1}$ \\ Mário César de Andrade ${ }^{1}$ \\ João Otacílio Libardoni dos Santos ${ }^{1}$
}

\title{
AVALIAÇÃO DA FORÇA MUSCULAR DE FLEXORES E EXTENSORES DE JOELHO EM INDIVÍDUOS COM E SEM OSTEOARTROSE
}

\author{
ASSESSMENT OF MUSCULAR STRENGTH OF KNEE FLEXORS AND \\ EXTENSORS IN INDIVIDUALS WITH AND WITHOUT OSTEOARTHRITIS
}

\begin{abstract}
RESUMO
O objetivo do estudo foi avaliar torques concêntrico e excêntrico de sujeitos com e sem osteoartrose de joelho. Especificamente: comparar o membro dominante e não dominante de ambos os grupos; comparar a relação concêntrica isquitibiais/quadríceps entre os grupos. O estudo foi aprovado pelo Comitê de Ética em Pesquisa da UDESC. Participaram 58 idosos, sendo 32 com osteoartrose de joelho e 26 saudáveis. Utilizou-se o dinamômetro isocinético KinCom. As coletas de dados realizaram-se, seguindo os seguintes procedimentos: 1)ficha cadastral; 2)antropometria (massa, estatura); 3) aquecimento e alongamento; 4)posicionamento dos indivíduos no equipamento com joelho em flexão de $90^{\circ}$ e estabilização da coxa; 5)familiarização dos sujeitos ao equipamento; 6)aquisição de três ciclos completos de contração concêntrica e excêntrica máxima, de flexão e extensão de joelho, a $60 \%$ s. Variáveis analisadas: pico de torque concêntrico e excêntrico de quadríceps e isquiotibiais, normalizados pela massa corporal e a relação pico de torque concêntrico de isquiotibiais/quadríceps de ambos os membros. Utilizou-se a estatística descritiva e não-paramétrica (Wilcoxon e Teste $U$ de Mann-Whitney) com $p \leq 0,05$. A maioria dos torques concêntricos e excêntricos foi maior no grupo controle, exceto os torques concêntricos de isquitibiais e excêntrico de quadríceps do membro não dominante. Os torques no membro dominante em indivíduos sem osteoartrose e os torques excêntricos de indivíduos com osteoartrose e a relação isquitibiais/quadríceps em indivíduos com osteoartrose foram maiores. Não foram encontradas diferenças estatisticamente significativas entre os praticantes e não praticantes de atividade física. Conclui-se que a osteoartrose provoca declínio de força muscular principalmente em quadríceps.
\end{abstract}

Palavras-chave: Osteoartrose; Torque; Força; Joelho.

\section{ABSTRACT}

The aim of this study was to evaluate concentric and eccentric torque in individuals with and without knee osteoarthritis, and who did and did not practice physical activity. Specifically: to compare concentric and eccentric torque between groups; to compare the torques of dominant and non-dominant limbs within groups; to compare the concentric hamstrings/quadriceps ratio between groups. Fifty-eight elderly people participated, 32 with knee osteoarthritis and 26 with healthy knees. An isokinetic KinCom dynamometer was used for data collection. Concentric and eccentric peak torques of the quadriceps and hamstrings, normalized for body weight and concentric peak torque hamstring/quadriceps ratio of both sides were the study variables. At the Biomechanics Laboratory the following procedures were carried out: 1) identification form; 2) anthropometry; 3) warm-up and stretching; 4) positioning of the individual in a seated position with knee flexion of $90^{\circ}$ and the back supported; 5) familiarization with the equipment; 6) acquisition of three reciprocal maximal concentric and eccentric contractions at $60 \%$ s. Descriptive statistics and the Wilcoxon and the U Mann-Whitney tests were used to analyze data, to $p \leq 0.05$. The majority of concentric and eccentric torques in the control group were higher than those in the knee osteoarthritis group, with the exception of the concentric torque of non-dominant hamstrings and the eccentric quadriceps of the non-dominant limb. Although not significant, dominance seems to be determinant of increased torques in individuals without knee osteoarthritis. No statistically significant differences were detected between those who did and those who did not take part in physical activity. The results demonstrate that osteoarthritis provokes decrease of force especially in quadriceps.

Key words: Osteoarthritis; Torque; Strength; Knee.

1. Universidade do Estado de Santa Catarina. Centro de Ciências da Saúde e do Esporte. Laboratório de Biomecânica. Florianópolis, SC. Brasil. 


\section{INTRODUÇÃO}

A Osteoartrose (OA) é uma doença degenerativa que atinge as articulações sinoviais e caracteriza-se por apresentar alterações na cartilagem articular, dando origem a zonas de fibrilação e fissuração, sendo observados também microfraturas, cistos, esclerose subcondrais e formação de osteófitos nas bordas $\operatorname{articulares}^{1,2}$. Embora com etiologia desconhecida, postula-se como possíveis causas etiológicas o fator sobrecarga, como fatores ocupacionais e sobrepeso, notoriamente em articulações de suporte de peso (joelho, quadril), provocando na patologia estabelecida dor articular protocinética, diminuição da força muscular, incapacidade funcional, alterações proprioceptivas e em fase mais avançada, deformidades.

A osteoartrose ocasiona a diminuição da força muscular e pode acarretar progressiva perda de função, pois os músculos são importantes absorvedores de choque que ajudam a estabilizar a articulação. Apesar de não acometer somente idosos, estudos apontam para a influência da idade na osteoartrose, neste sentido, estudos ${ }^{3}$ afirmam que a idade influencia a gravidade da osteoartrose, principalmente, em mulheres. $\mathrm{Na}$ osteoartrose de joelho (OAJ), observa-se precocemente diminuição de força de flexores e extensores de joelho ${ }^{4,5}$ que estão relacionadas à diminuição da capacidade funcional e a habilidade em realizar atividades como subir escadas, levantar, sentar e caminhar.

No entanto, apesar de estudos ${ }^{4-7}$ relacionarem a osteoartrose de joelho com a diminuição da força de flexores e extensores desta articulação e de estar bem documentado que a estabilidade dinâmica da articulação do joelho é dependente da manutenção da força muscular destes músculos, existe escassez de estudos brasileiros sobre os valores de torques concêntricos e excêntricos, bem como a relação isquiotibiais/ quadríceps em portadores de osteoartrose de joelho utilizando a dinamometria isocinética. A obtenção destes dados possibilitará a identificação de fatores geradores de incapacidade e a elaboração de tratamentos e exercícios mais apropriados a realidade de nossa população. Face às considerações anteriores realizou-se este estudo com o objetivo geral de avaliar os valores de pico de torque concêntrico e excêntrico de dois grupos de indivíduos: com e sem Osteoartrose de Joelho e, como objetivos específicos: a) comparar os torques concêntrico e excêntrico entre indivíduos com e sem osteoartrose; b) comparar os torques concêntrico e excêntrico entre os lados dominante e não dominante em indivíduos com e sem osteoartrose; c) comparar a relação Isquiotibiais/quadríceps entre indivíduos com e sem osteoartrose; d) comparar os torques concêntrico e excêntrico entre praticantes e não praticantes de atividade física em ambos os grupos; e) comparar a relação Isquiotibiais/quadríceps entre praticantes e não praticantes de atividade física em ambos os grupos.

\section{PROCEDIMENTOS METODOLÓGICOS}

Participaram deste estudo descritivo exploratório,
58 sujeitos, 32 portadores de OAJ (6 homens e 26 mulheres) e 26 indivíduos sem OAJ, na forma de grupo controle ( 6 homens e 20 mulheres), selecionados pelo processo não-probabilístico do tipo intencional, através de abordagem direta, tendo como critérios de inclusão: 1) para os indivíduos com osteoartrose de joelho diagnóstico confirmado pelos critérios do Colégio Americano de Reumatologia, idade superior a 50 anos, radiografia do joelho. 2) para os indivíduos sem osteoartrose, também idade superior a 50 anos, sem sintomas ou evidências radiológicas de osteoartrose de joelho.

A média de idade no grupo com OAJ foi de 63,13 anos $(s=7,60 \%, C V \%=12,04)$, semelhante ao grupo sem OAJ no qual se encontrou média de 65,42 anos $(s=7,64 \%, C V \%=11,67)$. Em relação aos dados antropométricos, o grupo com OAJ apresentou valores médios de massa de $78,29 \mathrm{Kg}(s=11,24, \mathrm{CV} \%=14,36)$ e estatura de $1,56 \mathrm{~cm}(s=0,08, C V \%=5,18)$, já para o grupo sem OAJ encontrou-se valores médios de massa de $65,82 \mathrm{Kg}(s=9,80, \mathrm{CV} \%=14,89)$ e estatura de $1,58 \mathrm{~cm}(s=0,05, C V \%=3,09)$.

Os sujeitos foram ainda classificados em praticantes e não praticantes de atividade física conforme a freqüência de prática, sendo considerado praticante os indivíduos que realizavam qualquer atividade física, no mínimo, 3 vezes semanais.

Utilizou como instrumentos de medida: a) Ficha cadastral com respectivos campos para registro das variáveis de identificação e antropométricas: idade, gênero, massa, estatura, prática de atividade física; b) Dinamômetro isocinético Kitec-Communicator (Kin-Com), modelo AP/MP e acessórios para flexão e extensão do joelho que acompanham o dinamômetro. A célula de carga do equipamento utilizado tem capacidade para medir forças entre 1 e $2000 \mathrm{~N}$ e resolução de $1 \mathrm{~N}$; c) balança com precisão de $100 \mathrm{~g}$; d) estadiômetro com escala de medida de $0,1 \mathrm{~cm}$.

Após aprovação pelo Comitê de Ética de Pesquisa da UDESC, processo $n^{\circ} 167 / 05$, realizaramse as coletas de dados no Laboratório de Biomecânica do CEFID/UDESC, adotando-se os seguintes procedimentos: a) preenchimento da ficha cadastral através de questionamento direto; b) pesagem dos sujeitos na balança, para normalização dos dados pela massa corporal (MC) e obtenção da estatura; c) aquecimento dos indivíduos através de caminhada em velocidade auto-elegida durante 5 minutos e alongamentos de flexores e extensores de joelho; d) posicionamento dos indivíduos no equipamento, sendo orientados a se posicionarem na cadeira regulável do dinamômetro, com o joelho em flexão de $90^{\circ}$, com a coxa estabilizada através de fita de contensão. $O$ eixo de rotação do dinamômetro foi alinhado com o epicôndilo femoral e a carga de resistência colocada cerca de $2 \mathrm{~cm}$ acima do maléolo; e) familiarização dos sujeitos ao equipamento, mediante a realização de vários ciclos completos de flexões e extensões de joelhos; f) aquisição propriamente dita, que consistiu em três ciclos completos de contração excêntrica e concêntrica máxima, nos movimentos de flexão e 
extensão de joelho, na velocidade de $60 \%$ s.

Selecionaram-se para estudo as variáveis: pico de torque normalizado (PTN) e a relação pico de torque concêntrico de isquiotibiais e quadríceps ${ }^{6}$. Com a finalidade de condensar o texto, as variáveis foram definidas por suas siglas, a saber: pico de torque concêntrico quadríceps dominante (PTCQD), pico de torque concêntrico quadríceps não-dominante (PTCQND), pico de torque concêntrico isquiotibiais dominante (PTCITD), pico de torque concêntrico de isquiotibiais não-dominante (PTCITND), pico de torque excêntrico quadríceps dominante (PTEQD), pico de torque excêntrico quadríceps não-dominante (PTEQND), pico de torque excêntrico isquiotibiais dominante (PTEITD) e pico de torque excêntrico isquiotibiais não-dominante (PTEITND), relação pico de torque concêntrico de isquiotibiais e quadríceps (IQT/QUA).

$\mathrm{Na}$ caracterização das variáveis, utilizou-se a estatística descritiva (média, desvio padrão e coeficiente de variação). Para comparar os valores de pico de torque e a relação concêntrica de IQT/QUA entre indivíduos com e sem OAJ e entre praticantes e não praticantes de atividade física utilizou-se a estatística não-paramétrica através do teste $U$ de Mann-Whitney. E para comparar os valores de torque entre o lado dominante e o não-dominante nos indivíduos com e sem osteoartrose utilizou-se o teste de Wilcoxon. Em todas as comparações adotou-se $p \leq 0,05$.

\section{RESULTADOS}

Os resultados estão apresentados de acordo com os objetivos específicos do trabalho. Assim, primeiramente são apresentadas as comparações dos torques concêntrico e excêntrico entre indivíduos com e sem osteoartrose, seguido das comparações entre os lados dominante e não dominante e pelas comparações das relações IQT/QUA entre indivíduos com e sem osteoartrose. Finalizou-se o capítulo com as comparações dos torques concêntrico e excêntrico e das relações IQT/QUA entre indivíduos praticantes e não praticantes de atividade física em ambos os grupos.

Inicialmente, fez-se a comparação entre os torques de indivíduos com e sem osteoartrose, cujos resultados estão dispostos na Tabela 1.

Analisando os resultados (Tabela 1), verificou-se que das oito variáveis comparadas entre os indivíduos com e sem osteoartrose de joelho, em apenas três foram encontrados valores significativamente menores: PTCQD, PTCQND e PTCITD, para o grupo com osteoartrose de joelho.

Na seqüência, compararam-se os dados entre os lados dominante (LD) e não dominante (ND), conforme pode ser visualizado na Tabela 2.

Analisando os resultados (Tabela 2), verificouse que para os indivíduos com osteoartrose de joelho (OAJ) os torques concêntricos e excêntricos foram maiores no lado dominante, exceto para a variável PTCQ. Nos indivíduos sem osteoartrose de joelho os valores médios de torques concêntricos e excêntricos foram maiores no lado dominante, embora não tenha sido encontrada diferença estatisticamente significativa em nenhum dos casos.

$\mathrm{Na}$ Tabela 3, a seguir, são apresentados os resultados das comparações das relações de picos de torque concêntrico IQT/QUA entre indivíduos com e sem osteoartrose.

Tabela 1. Comparação do Pico de Torque de quadríceps e isquiotibiais concêntrico e excêntrico entre indivíduos com e sem osteoartrose de joelho.

\begin{tabular}{ccccc}
\hline \multirow{2}{*}{ PTN (N.m/Kg) } & \multicolumn{2}{c}{$\bar{X}$} & & \\
\cline { 2 - 3 } & OAJ & Sem OAJ & & \\
\hline PTCQD & 44,10 & 78,17 & 4,292 & $0,000^{*}$ \\
PTCQND & 49,77 & 71,45 & 2,791 & $0,005^{*}$ \\
PTCITD & 37,59 & 47,90 & 2,197 & $0,028^{*}$ \\
PTCITND & 48,88 & 47,54 & 0,430 & 0,667 \\
PTEQD & 60,01 & 60,56 & 0,446 & 0,656 \\
PTEQND & 57,60 & 54,95 & 0,070 & 0,944 \\
PTEITD & 48,10 & 53,44 & 0,844 & 0,398 \\
PTEITND & 41,55 & 52,91 & 1,446 & 0,148 \\
\hline
\end{tabular}

* Valor significativo para os níveis pré-estabelecidos.

Tabela 2. Comparação do Torque de quadríceps e isquiotibiais concêntrico e excêntrico entre lado dominante e não dominante de indivíduos com e sem osteoartrose.

\begin{tabular}{cccccc}
\hline \multirow{2}{*}{ grupos } & PTN (N.m) & \multicolumn{2}{c}{$\bar{X}$} & Z & $\mathrm{p}$ \\
\cline { 3 - 4 } & & LD* $^{*}$ & ND $^{*}$ & & \\
\hline \multirow{2}{*}{ COM } & PTCQ & 44,10 & 49,77 & 1,734 & 0,083 \\
OAJ & PTCIT & 47,90 & 47,54 & 1,360 & 0,300 \\
& PTEQ & 60,01 & 57,60 & 0,692 & 0,489 \\
& PTEIT & 48,10 & 41,55 & 1,225 & 0,221 \\
& PTCQ & 78,17 & 71,45 & 1,171 & 0,241 \\
SEM & PTCIT & 47,90 & 47,54 & 1,036 & 0,300 \\
OAJ & PTEQ & 60,56 & 54,95 & 1,197 & 0,231 \\
& PTEIT & 53,44 & 52,91 & 0,417 & 0,677 \\
\hline
\end{tabular}

* LD: lado dominante; ND:lado não-dominante.

Tabela 3. Comparação da relação de pico de torque concêntrico IQT/QUA para o lado dominante e não dominante entre indivíduos com e sem osteoartrose.

\begin{tabular}{ccccc}
\hline \multirow{2}{*}{$\begin{array}{c}\text { Relação IQT/ } \\
\text { QUA (\%) }\end{array}$} & \multicolumn{2}{c}{$\bar{X}$} & \multirow{2}{*}{ Z } & $\mathrm{P}$ \\
\cline { 2 - 3 } & OAJ & Sem OAJ & & \\
\hline LD & 98,96 & 66,54 & 2,504 & $0,012^{*}$ \\
ND & 115,00 & 74,46 & 2,942 & $0,003^{*}$ \\
\hline
\end{tabular}

* Valor significativo para os níveis pré-estabelecidos

Analisando os resultados (Tabela 3), constataramse diferenças estatisticamente significativas entre 
o lado dominante e não dominante em ambos os grupos, sendo que os indivíduos com osteoartrose apresentaram valores da relação IQT/QUA maiores, tanto para o lado dominante quanto para o lado não dominante.

Ao se considerar a prática de atividade física, fezse a comparação entre os praticantes e não praticantes de atividade física e os resultados estão apresentados na Tabela 4, a seguir.

Tabela 4. Comparação do pico de torque de quadríceps e isquiotibiais concêntrico e excêntrico entre praticantes e não praticantes de atividade física de indivíduos com e sem osteoartrose.

\begin{tabular}{|c|c|c|c|c|c|}
\hline & \multirow[t]{2}{*}{ Variáveis } & \multicolumn{2}{|c|}{$\bar{X}$ (N.m) } & \multirow[t]{2}{*}{ Z } & \multirow[t]{2}{*}{$p$} \\
\hline & & PAF & NPAF & & \\
\hline \multirow{7}{*}{ 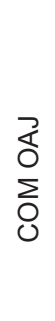 } & PTCQD & 40,61 & 49,21 & $-0,729$ & 0,466 \\
\hline & PTCQND & 50,56 & 48,62 & $-1,458$ & 0,145 \\
\hline & PTCITD & 41,33 & 32,12 & $-1,900$ & 0,057 \\
\hline & PTCITND & 54,80 & 40,23 & $-1,497$ & 0,134 \\
\hline & PTEQD & 60,69 & 59,01 & $-0,173$ & 0,863 \\
\hline & PTEQND & 67,55 & 43,05 & $-0,902$ & 0,367 \\
\hline & PTEITD & 50,09 & 45,20 & $-0,480$ & 0,631 \\
\hline \multirow{9}{*}{ 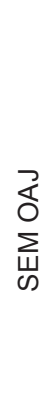 } & PTEITND & 45,70 & 35,48 & $-0,902$ & 0,367 \\
\hline & PTCQD & 87,73 & 65,14 & $-0,677$ & 0,498 \\
\hline & PTCQND & 78,97 & 61,19 & $-1,082$ & 0,279 \\
\hline & PTCITD & 56,51 & 36,15 & $-1,714$ & 0,086 \\
\hline & PTCITND & 62,38 & 27,30 & $-1,579$ & 0,114 \\
\hline & PTEQD & 68,83 & 49,27 & $-0,068$ & 0,946 \\
\hline & PTEQND & 56,40 & 52,97 & $-0,653$ & 0,514 \\
\hline & PTEITD & 59,12 & 44,44 & 0,000 & 1,000 \\
\hline & PTEITND & 60,21 & 44,22 & $-0,541$ & 0,588 \\
\hline
\end{tabular}

* Valor significativo para os níveis pré-estabelecidos.

Analisando os resultados das comparações (Tabela 4) não foram constatadas diferenças estatisticamente significativas para todas as variáveis comparadas entre os indivíduos praticantes e não praticantes de atividade física nos grupos com e sem osteoartrose de joelho. Embora não tenham sido constatadas diferenças significativas entre os grupos, verificou-se que das oito variáveis comparadas somente em uma delas: pico de torque concêntrico de quadríceps dominante (PTCQD), os indivíduos com osteoartrose não praticantes de atividade física apresentaram valores médios maiores $(49,21)$. Verificou-se ainda nas oito variáveis comparadas, valores médios consideravelmente maiores em indivíduos praticantes de atividade física para o grupo controle.

Por fim, compararam-se as relações de picos de torque concêntrico IQT/QUA entre praticantes e não praticantes de atividade física nos grupos de indivíduos com e sem osteoartrose de joelho.

Ao observar os resultados da Tabela 5, não foram constatadas diferenças estatisticamente significativas para a relação IQT/QUA entre praticantes e não praticantes de atividade física em ambos os grupos, de indivíduos com e sem osteoartrose de joelho. Contudo, foram obtidos valores médios da relação IQT/QUA consideravelmente maiores nos indivíduos praticantes de atividade física tanto com como sem osteoartrose para o lado dominante e não dominante.

Tabela 5. Comparação da relação de pico de torque concêntrico IQT/QUA para praticantes e não praticantes de atividade física entre indivíduos com e sem osteoartrose.

\begin{tabular}{lccccc}
\hline \multirow{2}{*}{ grupo } & \multirow{2}{*}{$\begin{array}{c}\text { variá- } \\
\text { veis }\end{array}$} & \multicolumn{2}{c}{$\bar{X}(\mathrm{~N} . \mathrm{m})$} & \multirow{2}{*}{$\mathrm{z}$} & $\mathrm{p}$ \\
\cline { 3 - 4 } & & PAF & NPAF & & \\
\hline COM & LD & 113,69 & 74,26 & $-1,094$ & 0,274 \\
OAJ & ND & 124,14 & 103,10 & $-0,307$ & 0,757 \\
SEM & LD & 74,44 & 62,21 & $-1,261$ & 0,207 \\
OAJ & ND & 90,54 & 60,66 & $-0,788$ & 0,431 \\
\hline
\end{tabular}

LD: lado dominante; ND:lado não-dominante. PAF: indivíduos praticantes de atividade física; NPAF: indivíduos não praticantes de atividade física.

\section{DISCUSSÃO}

$\mathrm{Na}$ comparação dos torques de isquiotibiais e quadríceps concêntrico e excêntrico entre indivíduos com e sem osteoartrose de joelho, constatou-se que os indivíduos do grupo sem osteoartrose, conforme o esperado, apresentaram valores médios de pico de torque superiores na maioria das variáveis, exceto PTCITND e PTEQND (Tabela 1). Entretanto, houve diferença estatisticamente significativa apenas nos torques CQD, CQND, CITD ( $p=0,000 ; 0,005 ; 0,028$, respectivamente). Tais resultados, com diminuição do torque, assemelham-se a outros estudos ${ }^{4,5,7-9}$. Dentre estes, encontrou-se diminuição do torque excêntrico e concêntrico de quadríceps e isquiotibiais em indivíduos com OAJ, quando comparados a indivíduos saudáveis ${ }^{4}$. Os autores ${ }^{4}$ acrescentam ainda que o torque excêntrico parece ser menos afetado pela patologia do que o torque concêntrico.

A osteoartrose de joelho está associada a $50-60 \%$ da redução do torque máximo do quadríceps ${ }^{7}$, possivelmente, resultado da atrofia por desuso e inibição artrogênica. Já em outro estudo cita-se que a diminuição da força de quadríceps encontra-se em torno de 30 a $50 \%$ em indivíduos portadores de osteoartrose quando comparados a indivíduos saudáveis dentro da mesma faixa etária ${ }^{5}$. Os resultados encontrados no presente estudo para os torques concêntricos de quadríceps e em ambos os membros corroboram, com tais estudos.

Em outro estudo ${ }^{10}$, é afirmado que a dor e a rigidez musculares apresentaram-se como principais determinantes das diferenças de torque e trabalho isocinético em pacientes com OA. Entretanto, mesmo que estudos comprovem a diminuição de força 
muscular nesses indivíduos ${ }^{12}$, ainda não está claro se a extensão da fraqueza do músculo está relacionada à dor crônica, ao desuso ou à atrofia do músculo.

Contemplando o segundo objetivo do estudo, fez-se a comparação dos valores de torques entre os lados dominantes e não dominantes. Verificou-se então que no grupo com osteoartrose a maioria das variáveis apresentou valores médios superiores para o lado dominante, exceto para o torque de quadríceps concêntrico (Tabela 2). Entretanto, estas diferenças não foram estatisticamente significativas em todas as variáveis estudadas. O resultado encontrado neste estudo pode ser atribuído a uma particularidade metodológica, pois não foi controlado o grau de osteoartrose no lado dominante e não dominante, o que pode ter influenciado os valores de torque.

No grupo de indivíduos sem osteoartrose, verificaram-se valores superiores de picos de torque concêntrico e excêntrico de flexores e extensores no lado dominante, entretanto, estas diferenças não foram significativas. Valores semelhantes foram obtidos no lado dominante em alguns estudos ${ }^{8,12}$, contudo, em outro estudo ${ }^{13}$ com idosas saudáveis não foi encontrado diferença significativa entre o lado dominante e não dominante, por isso, autores ${ }^{4}$ consideram que a diferença de torque entre segmento dominante e nãodominante é fisiológica.

A relação IQT/QUA é um parâmetro comumente usado para descrever o equilíbrio muscular da articulação do joelho. Comparando-se esta relação de pico de torque concêntrico IQT/QUA em indivíduos com e sem OAJ, constatou-se valores significativamente maiores em indivíduos com OAJ, o que sugere maior desequilíbrio de força muscular entre flexores e extensores, sendo que, geralmente, a diminuição de força de quadríceps ocorre de forma mais acentuada que a dos isquiotibiais. E este desequilíbrio de forças pode causar alterações funcionais que aceleram a degeneração articular e/ou causam incapacidade funcional.

Alguns estudos ${ }^{6,14}$ apontam que a relação IQT/ QUA tende a ser aproximadamente $60 \%$ a $76 \%$ em adultos saudáveis. Considerando estes valores, a relação IQT/QUA no presente estudo, nos indivíduos sem OA estaria dentro do normal. Entretanto, os valores desta relação em indivíduos com OA denotam uma redução acentuada do torque de quadríceps em relação ao torque de isquiotibiais, com valores de 98 a $115 \%$. Em outro estudo com idosos ${ }^{15}$ essa relação também foi significativamente aumentada no grupo com osteoartrose, em função da maior força de quadríceps em relação à de isquiotibiais.

Na comparação do pico de torque de quadríceps e isquiotibiais concêntrico e excêntrico entre praticantes e não praticantes de atividade física em indivíduos com e sem osteoartrose de joelho, não foram identificadas diferenças estatisticamente significativas. Contudo, os indivíduos praticantes de atividade física de ambos os grupos apresentaram valores médios maiores para quase todas as variáveis analisadas, o que evidencia a importância da prática de atividade física na manutenção da força muscular.

Embora estudos confirmem o incremento de força muscular de extensores e flexores do joelho de idosos decorrentes da prática da atividade física ${ }^{16}$, principalmente em programas relacionados ao treinamento de resistência muscular, o presente estudo não verificou diferença significativa, o que pode ser atribuído a uma particularidade metodológica em que não se controlou o tipo de atividade física praticada, apenas a freqüência. Tais achados vão ao encontro dos resultados de estudo ${ }^{15}$ em que, sob as mesmas condições, foi realizada a comparação das variáveis de força muscular entre idosos ativos e sedentários e também não foi encontrado diferença significativa.

Em complemento, foi verificado que, para idosos saudáveis apenas duas sessões por semana de treinamento geral composto por atividades da vida diária não são suficientes para induzir alterações que promovam um acréscimo sobre a força da musculatura dos membros inferiores. Já um treinamento específico para essa capacidade física torna-se eficiente ${ }^{12,17}$. Tais afirmações demonstram que além da freqüência de prática de atividade física o tipo de treinamento parece ter uma implicação direta na percepção de ganhos de força muscular.

\section{CONCLUSÃO}

Diante dos resultados apresentados e com base no referencial teórico consultado, concluiu-se que: a) A osteoartrose produziu redução dos valores de torque concêntrico e excêntrico de flexores e extensores do joelho, com exceção para os torques concêntricos de isquiotibiais não dominantes e excêntricos de quadríceps não dominante; b) A dominância lateral parece ser determinante no aumento dos torques nos indivíduos sem osteoartrose, c) Para os indivíduos sem osteoartrose, os valores da relação IQT/QUA enquadram-se dentro dos valores de normalidade apresentados na literatura, entretanto, indivíduos com OA apresentaram valores superiores, denotando perda de força importante em quadríceps, este desequilíbrio de força pode gerar importante repercussões funcionais, sugerindo estudos que confirmem estes resultados e detectem suas repercussões. d) A prática de atividade física não produziu diferenças significativas no torque em ambos os grupos, entretanto, este estudo não controlou nem a modalidade nem o tipo de treinamento realizado, o que pode justificar este achado.

\section{REFERÊNCIAS BIBLIOGRÁFICAS}

1. Coimbra IB, Pastor EH, Greve JMA, Puccinelli MLC, Fuller R, Cavalcanti FS, et al. Consenso brasileiro para o tratamento da osteoartrose. Rev Bras Reum 2002;42(6):371-374.

2. Marques AP, Kondo A. A fisioterapia na osteoartrose: uma revisão de literatura. Rev Bras Reum 1998;38(2):83-90.

3. Caspi D, Flusser G, Farber I, Ribak J, Leibovitz A, Habot B, et al. Clinical, Radiologic, Demographic, and 
Occupational Aspects of Hand Osteoarthritis in the Elderly. Semin Arthritis Rheum 2001;30(5):321-331.

4. Gür H, Çakin N. Muscle Mass, Isokinetic Torque, and Functional Capacity in Women With Osteoarthritis of the Knee. Arch Phys Med Rehabil 2003;84(10):1534-1541.

5. Fisher NM, Pendergast DR, Gresham GE, Calkins E. Muscle rehabilitation: its effect on muscular and functional performance of patients with knee osteoarthritis. Arch Phys Med Rehabil 1991;72(6):367-374.

6. Arantes PM, Alencar MA, Machala CC, Faria JC, Camargos FFO, Dias JMD, et al. Valores isocinéticos de referência para força muscular, trabalho dos flexores e extensores do joelho e relação isquiotibiais/quadríceps de mulheres idosas. Anais do X Congresso Brasileiro de Biomecânica. Ouro Preto: UFMG; 2003.p.330-334.

7. Hassan BS, Mockett S, Dohert M. Static postural sway proprioception, and maximal voluntary quadriceps contraction in patients with knee osteoarthritis and normal control subjects. Ann Rheum Dis 2001;60:612-618.

8. Tan J, Balci N, Sepici V, Gener FA. Isokinetic and isometric strength in osteoarthritis of the knee. Am J Phys Med Rehabil 1995;74(5):364-369.

9. Hurley MV, Scott DL, Rees J, Newham J. Sensoriomotor changes and functional performance in the patients with knee osteoarthritis. Ann Rheum Dis 1997;56(11):641-648.

10. Hassan BS, Doherty SA, Mockett S, Doherty M. Effect of pain reduction on postural sway, proprioception, and quadriceps strength in subjects with knee osteoarthritis. Ann Rheum Dis 2002;61(5):422-428.

11. Emrani A, Bagheri H, Hadian MR, Jabal-Ameli
M, Olyaei GR, Talebian S. Isokinetic strength and functional status in knee osteoarthritis. J Phys Ther Sci 2006;18(2):107-114.

12. Carvalho J, Oliveira J, Magalhães J, Ascensão A, Mota J, Soares JM da. Força muscular em idosos II — Efeito de um programa complementar de treino na força muscular de idosos de ambos os sexos. Rev Port Cien Desp 2004;4(1):58-65.

13. Aquino M de, Leme LEG, Amatuzzi MM, Greve, JMD, Terreri ASAP, Andrusaitis FR, et al. Isokinetic assessment of knee flexor/extensor muscular strength in elderly women. Rev Hosp Clín 2002;57(4):131-134.

14. Croce RV, Pitetti KH, Horvat M, Miller J. Peak torque, average power, and hamstrings/quadriceps ratio in nondisabled adults and adults with mental retardation. Arch Phys Med Rehabil 1996;77(4):369-372.

15. Zacaron KAM, Dias JMD, Abreu NS, Dias RC. Nível de atividade física, dor e edema e suas relações com a disfunção muscular do joelho de idosos com osteoartrite. Rev Bras Fisioter 2006;10(3):279-284.

16. Carvalho J, Oliveira J, Magalhães J, Ascensão A, Mota J, Soares JM da. Efeito de um programa de treino em idosos: comparação da avaliação isocinética e isotônica. Rev bras educ fis esp 2003;17(1):74-84.

17. Carvalho J, Oliveira J, Magalhães J, Ascensão A, Mota J, Soares J M da. Força muscular em idosos I - Será o treino generalizado suficientemente intenso para promover o aumento da força muscular em idosos de ambos os sexos? Rev Port Cien Desp 2004;4(1):51-57.

\section{Endereço para Correspondência}

Juliane de Oliveira

Recebido em 07/04/08

Laboratório de Biomecânica

Rua Paschoal Simone, 358 - Coqueiros

Revisado em 29/05/08

CEP 88080-350 - Florianópolis - SC.

Aprovado em 27/06/08

E-mail: julifisioterapia@gmail.com 